\title{
Total knee replacement after high tibial osteotomy: time-to-event analysis and predictors
}

\author{
Codie A. Primeau MSc, Trevor B. Birmingham PhD, Kristyn M. Leitch PhD, Kevin R. Willits MD, \\ Robert B. Litchfield MD, Peter J. Fowler MD, Jacquelyn D. Marsh PhD, Bert M. Chesworth PhD, \\ Stephanie N. Dixon PhD, Dianne M. Bryant PhD, J. Robert Giffin MD MBA
}

- Cite as: CMAJ 2021 February 1;193:E158-66. doi: 10.1503/cmaj.200934

\begin{abstract}
BACKGROUND: An important aim of high tibial osteotomy (HTO) is to prevent or delay the need for total knee replacement (TKR). We sought to estimate the frequency and timing of conversion from HTO to TKR and the factors associated with it.
\end{abstract}

METHODS: We prospectively evaluated patients with osteoarthritis (OA) of the knee who underwent medial opening wedge HTO from 2002 to 2014 and analyzed the cumulative incidence of TKR in July 2019. The presence or absence of TKR on the HTO limb was identified from the orthopedic surgery reports and knee radiographs contained in the electronic medical records for each patient at London Health Sciences Centre. We used cumulative incidence curves to evaluate the primary outcome of time to TKR. We used multivariable Cox proportional hazards analysis to assess potential preoperative predictors including radiographic disease severity, malalignment, correction size, pain, sex, age, body mass index (BMI) and year of surgery.

RESULTS: Among 556 patients who underwent $643 \mathrm{HTO}$ procedures, the cumulative incidence of TKR was 5\% (95\% confidence interval $[\mathrm{Cl}] 3 \%-7 \%$ ) at 5 years and $21 \%$ (95\% Cl 17\%-26\%) at 10 years. With the Cox proportional hazards multivariable model, the following preoperative factors were significantly associated with an increased rate of conversion: radiographic OA severity (adjusted hazard ratio [HR] $1.96,95 \% \mathrm{Cl} 1.12-3.45)$, pain (adjusted HR 0.85, 95\% Cl 0.75-0.96)], female sex (adjusted HR 1.67, 95\% Cl 1.082.58), age (adjusted HR 1.50 per $10 \mathrm{yr}, 95 \%$ $\mathrm{Cl}$ 1.17-1.93) and BMI (adjusted HR 1.31 per $\left.5 \mathrm{kng} / \mathrm{m}^{2}, 95 \% \mathrm{Cl} 1.12-1.53\right)$.

INTERPRETATION: We found that $79 \%$ of knees did not undergo TKR within 10 years after undergoing medial opening wedge HTO. The strongest predictor of conversion to TKR is greater radiographic disease at the time of HTO.
T he burden of knee osteoarthritis (OA) on patients and health care systems is substantial and growing. ${ }^{1}$ The current treatment strategy that relies largely on total knee replacement (TKR) for end-stage disease may not be sustainable..$^{2-5}$ Reduced quality of life and loss of productivity due to knee $\mathrm{OA}$ in middle-aged people in the workforce is particularly problematic..$^{5-8}$ The global prevalence of knee OA peaks at about 50 years of age. ${ }^{9}$ Worldwide, the estimated years lived with disability is 2.4 million for people younger than 50 years of age, the approximate age of peak prevalence for knee OA. ${ }^{9,10}$ Accordingly, the number of middle-aged patients seeking treatment for knee OA, including TKR, is increasing. ${ }^{11}$ Joint replacement may not be the most appropriate treatment for these patients. ${ }^{12}$ Earlier TKR is associated with prosthesis infection, ${ }^{13}$ lower patient satisfaction ${ }^{14}$ and revision surgery; ${ }^{15-18}$ about
$25 \%$ of all TKRs are considered "likely inappropriate." ${ }^{19}$ Clinicians have identified a clear treatment gap between exhausting nonoperative management and appropriateness for TKR, resulting in years of pain, decreased function, productivity losses and associated costs. $^{5-9,20,21}$ It is therefore imperative to identify additional effective treatments for the large group of patients with knee OA.

Medial opening wedge high tibial osteotomy (HTO) is a limb realignment surgery intended for patients with medial compartment knee OA who are not suitable candidates for TKR because of less severe disease, younger age and greater physical demands. The purpose of HTO is to correct malalignment, thereby shifting load away from the more involved knee compartment and limit OA progression. ${ }^{22,23}$ Substantial shifts in knee loading ${ }^{24,25}$ have resulted in clinically important improvements in pain and function 
after $\mathrm{HTO}^{26,27}$ and the procedure is cost-effective, ${ }^{28,29}$ yet the surgery is uncommon in Canada. ${ }^{30}$ Unlike the high and increasing rates of other knee surgical procedures including arthroscopy ${ }^{31}$ and $\mathrm{TKR},{ }^{32}$ rates of $\mathrm{HTO}$ remain low. ${ }^{33,34}$

High tibial osteotomy may help fill the treatment gap between nonsurgical treatments and definitive TKR. At the London Health Sciences Centre in London, Ontario, HTO is performed frequently with a goal of preventing or delaying the need for TKR. Thus, it is appropriate to investigate the duration of benefit of HTO, and the preoperative characteristics associated with it. When quantified as conversion from HTO to TKR, registries using administrative data enable large sample sizes ( $>2500$ patients) to estimate cumulative incidence of TKR. ${ }^{30,35,36}$ However, there can be limitations in using only administrative data, including confirming the correct procedure, limb and diagnosis. Administrative data often lack detailed information assessed preoperatively, such as radiographic features (e.g., disease severity and lower limb alignment) and patient-reported outcome measures. Previously reported predictors of conversion to TKR such as female sex and greater age ${ }^{30,35-39}$ may be influenced (perhaps confounded) by other clinical characteristics not typically included in administrative data. Therefore, our objective was to investigate the cumulative incidence of TKR after medial opening wedge HTO and potential predictors using data collected prospectively from a single Canadian centre that focuses on HTO. Specifically, we evaluated the time to conversion from HTO to TKR and investigated the association of HTO preoperative characteristics with subsequent TKR.

\section{Methods}

\section{Study design, data sources and population}

We recruited patients from the London Health Sciences Centre, a large academic teaching hospital providing clinical services to residents in Southwestern Ontario. Among other clinics, the Orthopedics Program at the centre includes the Fowler Kennedy Sport Medicine Clinic and the Rorabeck Bourne Joint Replacement Clinic, located on the campus of Western University; about 1600 hip and knee replacements are performed each year. We prospectively evaluated patients referred to the Wolf Orthopaedic Biomechanics Laboratory, Fowler Kennedy Sport Medicine Clinic between 2002 and 2014. We followed patients from their time of enrolment until July 31,2019 , providing a minimum potential follow-up of 5 years from the time of their HTO.

Four surgeons participated. At study initiation, 2 were early in independent practice and 2 were well-established; all of the surgeons had completed international fellowships in orthopedic sports medicine. All performed a medial opening wedge HTO technique similar to one previously described in detail 27,40 and summarized in Appendix 1 (available at www.cmaj.ca/lookup/ doi/10.1503/cmaj.200934/tab-related-content).

Patients were referred by other orthopedic surgeons and primary care physicians because of unresolved knee pain or reduced function, and dissatisfaction with the level of improvement achieved with nonoperative treatment. Patients underwent an examination completed by a surgeon that included radiography of the knee, specifically a full-limb (hip-to-ankle) standing anteroposterior view, to enable the assessment of frontal plane alignment using the mechanical axis angle (MAA). ${ }^{41,42}$ Appropriate candidates for HTO had varus alignment (MAA $<0^{\circ}$ ), radiographic features of $\mathrm{OA}$ in the medial tibiofemoral compartment and pain confined primarily to the medial knee (identified by the patient during examination). Patients with lateral compartment or patellofemoral features of OA were candidates for HTO as long as pain and radiographic changes were most severe in the medial compartment. Patients with end-stage radiographic OA in 2 or more knee compartments were referred to the affiliated arthroplasty clinic because they were considered poor candidates for HTO.

\section{Outcome and assessment}

Our primary outcome was time to conversion from HTO to TKR. For each patient who underwent HTO in our cohort, we identified the presence or absence of TKR using the operative reports and patient radiographs located in the electronic medical record system of the centre. The operative report serves as the official record of the surgery and includes pertinent procedural details. For our study, when there was a record of TKR we identified the surgery date and limb operated on, then viewed postoperative knee radiographs to confirm TKR on the HTO limb. When there was no record of TKR, we examined knee radiographs from the patient's last HTO follow-up visit to confirm the absence of TKR. Follow-up time was censored at the time of last contact or at death, as appropriate. ${ }^{43}$

\section{Potential predictors}

The radiographic assessments and the preoperative value for patient-reported outcome measures were obtained within 12 weeks of HTO. Clinical characteristics included the KellgrenLawrence $(\mathrm{KL})$ grade of radiographic OA severity before $\mathrm{HTO},{ }^{44}$ preoperative MAA ${ }^{41,42}$ and size of osteotomy correction (in $\mathrm{mm}$ ). In addition, we included the Knee injury and Osteoarthritis Outcome Score (KOOS) pain subscale. ${ }^{45}$ We also included demographic variables such as age, sex and body mass index (BMI) because they have been reported to be predictors of conversion to TKR..$^{30,35-39,46-48}$ Finally, we included HTO surgery date to account for potential variations or changes in practice over time.

\section{Statistical analysis}

We performed a Kaplan-Meier analysis and generated life tables with $95 \%$ confidence intervals (Cls) to estimate cumulative incidence as a function of time for the conversion from HTO to TKR. We also performed subgroup Kaplan-Meier analyses for radiographic disease severity and sex. A competing risk analysis was not required because the rate of death was low in our sample population.

We fitted a multivariable Cox proportional hazards model to estimate hazard ratios (HR) and $95 \% \mathrm{Cls}$ for time to conversion from HTO to TKR. We selected potential predictor variables a priori to minimize the risk of model overfitting. ${ }^{49}$ Specific variables 
included radiographic OA severity (i.e., $\mathrm{KL}$ grade divided as $\mathrm{KL} 2$ or below v. KL 3 or 4), MAA, size of correction (in $\mathrm{mm}$ ), baseline knee pain (i.e., KOOS pain score per 10 points), sex, age (per 10 years), BMI (per $5 \mathrm{~kg} / \mathrm{m}^{2}$ ) and year of surgery.

We evaluated the proportional hazards assumption both graphically and statistically, using log-log curves and Schoenfeld residuals. Because some patients had HTO performed on both knees, we adjusted the variance at the patient level for clustering among bilateral procedures by incorporating a robust sandwich estimator in the model to ensure appropriate type 1 error rates. We also completed a sensitivity analysis that included only the first HTO in those patients.

We conducted 2 post hoc analyses. We repeated the Cox proportional hazards model analysis while stratifying by surgeon and also explored the effect of correction angle achieved with surgery by repeating the primary Kaplan-Meier analysis for 3 subgroups based on the postoperative MAA $\left(<0^{\circ}\right.$ [varus], $0^{\circ}$ to $3^{\circ}$ [neutral] and $>3^{\circ}$ [valgus]). We also repeated the Cox model while adding the categorical correction angle variable. We performed all analyses using Stata 16 (StataCorp) statistical software using a 2 -sided $p$ value $<0.05$ to indicate statistical significance.

\section{Ethics approval}

This study was approved by Western University's Research Ethics Board for Health Sciences Research Involving Human Subjects.

\section{Results}

We screened 725 patients' knees for potential inclusion. Our final cohort included 643 knees in 556 patients who underwent

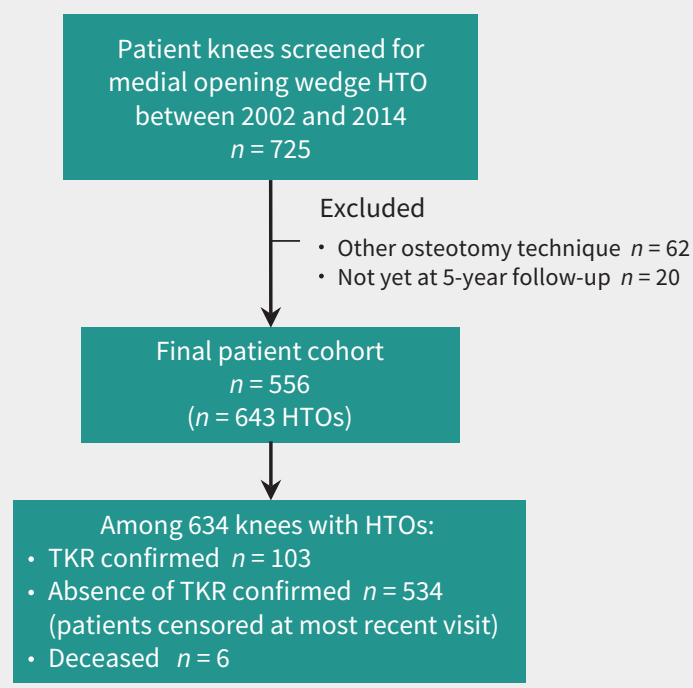

Figure 1: Flow diagram for patient selection. Note: HTO = high tibial osteotomy, TKR = total knee replacement. medial opening wedge HTO, including 87 staged bilateral procedures (Figure 1). Most patients in our cohort were male, middle-aged, overweight, had varus alignment, substantial knee pain and moderate radiographic OA severity in the medial tibiofemoral compartment (Table 1). ${ }^{50}$ One hundred and three TKRs (16.0\%) were performed, and 6 patients (1.1\%) died during follow-up. Of the 87 patients who underwent staged bilateral osteotomies, 15 underwent subsequent staged bilateral TKR. The median follow-up time was 7 (interquartile range [IQR] 4-10) years.

The cumulative incidence of conversion to TKR after HTO was $5 \%$ at 5 years and $21 \%$ at 10 years (Figure 2); this suggests that $95 \%$ of knees did not undergo TKR within 5 years of HTO and $79 \%$ did not undergo TKR within 10 years. Subgroup analyses based on radiographic severity and sex are illustrated in Figure 3 and Figure 4 . We found that cumulative incidence was $2 \%$ at 5 years and $13 \%$ at 10 years for patients with early-stage disease severity $(K L \leq 2)$. Cumulative incidence was $7 \%$ at 5 years and $32 \%$ at 10 years for female patients. Life tables are presented in Appendix 2, Supplementary Tables 1-3, available at www.cmaj. ca/lookup/doi/10.1503/cmaj.200934/tab-related-content.

We determined that greater radiographic disease, greater knee pain, female sex, greater age and higher BMI were all associated with an increased rate of conversion to TKR (Table 2). Greater radiographic disease severity was the strongest predictor (adjusted HR 1.96, 95\% Cl 1.12-3.45), which suggests that at any point in time, patients with later-stage disease at time of HTO are more than twice as likely to have TKR compared with patients with earlier-stage disease.

We found that interpretation of results was similar when the primary analysis was repeated after including only the first procedure for patients who underwent bilateral procedures (Appendix 2, Supplementary Table 4), and when stratifying by surgeon (post hoc; Appendix 2, Supplementary Table 5). Post hoc analyses suggested the 5-year and 10-year cumulative risk of conversion to TKR, respectively, were $9 \%$ and $30 \%$ for HTO correction angles less than $0^{\circ}, 2 \%$ and $14 \%$ for angles $0^{\circ}-3^{\circ}$, and $6 \%$ and $23 \%$ for angles greater than $3^{\circ}$ (Appendix 2, Supplementary Table 6). Compared with $0^{\circ}-3^{\circ}$ (reference), we found that correction angles less than $0^{\circ}$ were associated with increased rate of TKR (adjusted HR 1.87, 95\% Cl 1.18-3.00). Although our results suggested correction angles greater than $3^{\circ}$ could also be associated with an increased rate of TKR (adjusted HR 1.40, $95 \% \mathrm{Cl} \mathrm{0.80-2.46),} \mathrm{the} \mathrm{confidence} \mathrm{interval} \mathrm{included} 1$ (Appendix 2, Supplementary Table 7).

\section{Interpretation}

We estimated the cumulative incidence of conversion from medial opening wedge HTO to TKR at 10 years to be $21 \%$ using a time-to-event analysis. Our findings suggest overall longevity of medial opening wedge HTO and do not imply that TKR is a subsequent complication or failure. Our findings differ only slightly from rates reported in international studies (Finland [27\%] and Sweden [30\%]) and are slightly lower than rates found in provincial studies (Ontario, 33\%) that used administrative data. ${ }^{30,35,36}$ 


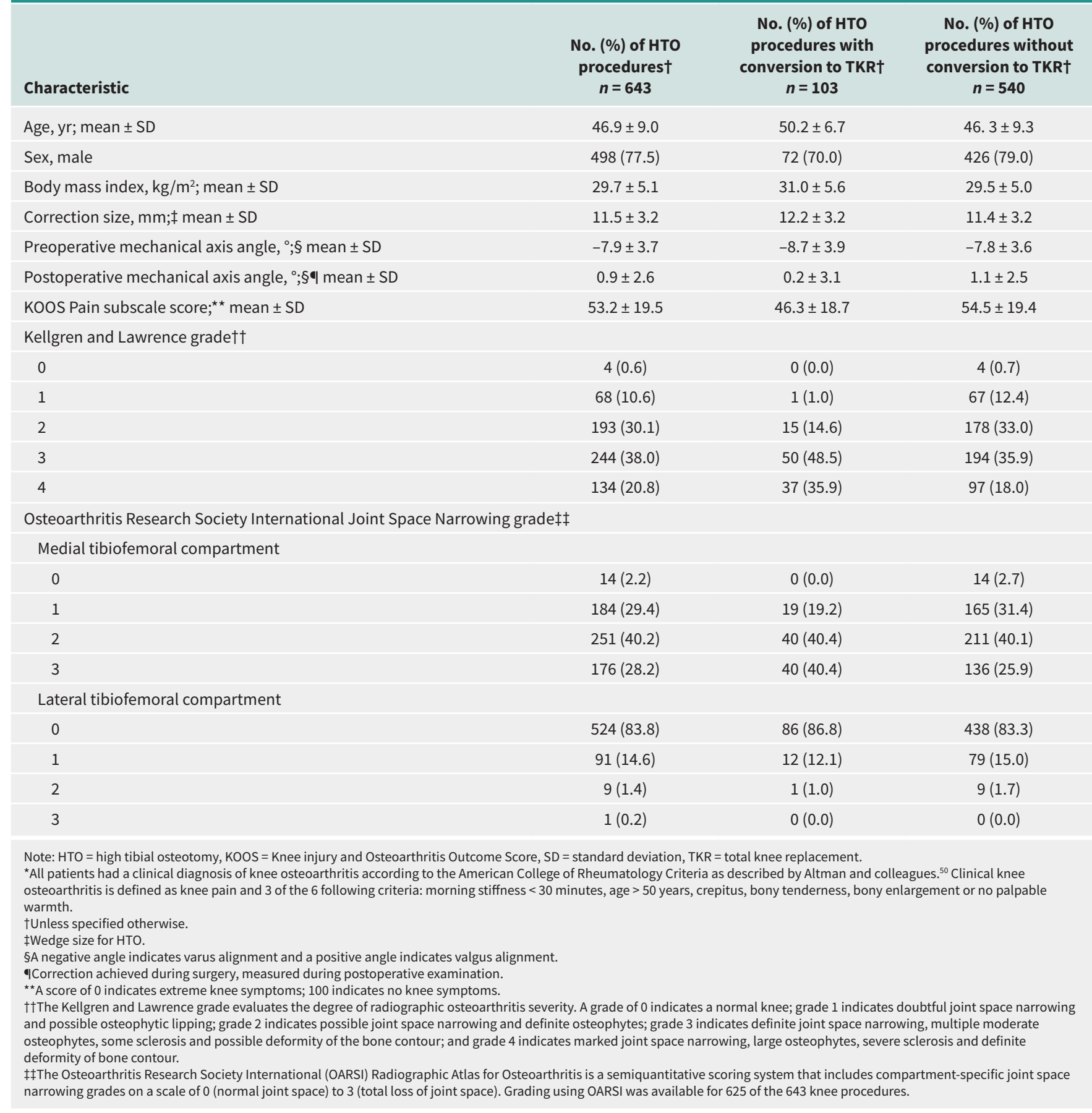

Differences in the accuracy of administrative versus clinical data may explain this slight variation. Specifically, the previously reported rate of TKR after HTO in Ontario (33\%) was described as potentially overestimated owing to the inability to match the TKR limb with the HTO limb in the administrative data set. ${ }^{30}$ Alternatively, it is possible that our observed rate $(21 \%)$ is underestimated if some patients sought TKR outside of London after their study censoring date. Other possible explanations for the differences between these reported rates include the earlier (1994-2010) ${ }^{30}$ versus later (2002-2014) time periods studied, referral network, surgical technique and rehabilitation. The present cumulative incidence of TKR of 5\% at 5 years and $21 \%$ at 10 years after HTO are consistent with previous smaller cohort studies that reported rates of $1 \%-20 \%$ at 5 years $^{37,51-55}$ and $8 \%-35 \%$ at 10 years. ${ }^{37,52,55-57}$

We found that radiographic severity of knee $O A$ at the time of medial opening wedge HTO was the strongest predictor of conversion to TKR (Figure 3 and Table 2), which is consistent 


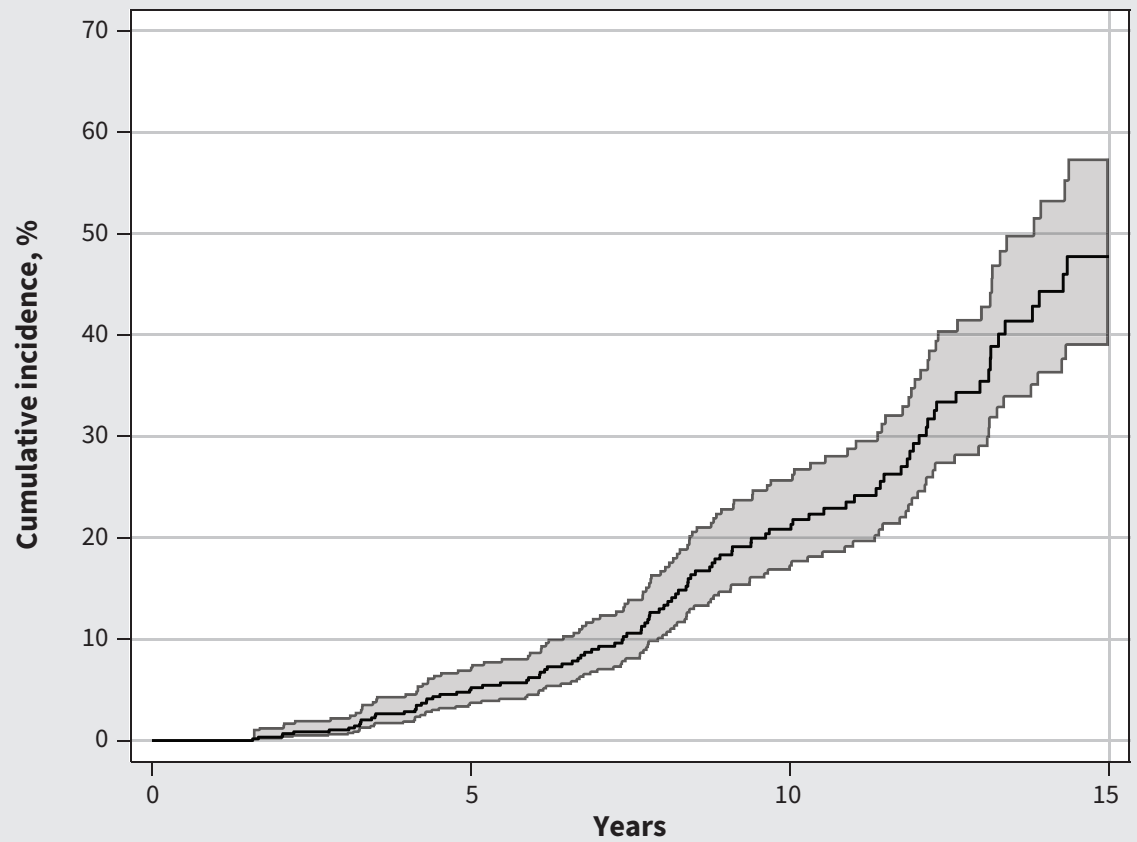

Number of patient knees at risk

Figure 2: Cumulative incidence curve (with 95\% confidence intervals as shaded areas) for patients undergoing 1 or more high tibial osteotomies ( $n=643 \mathrm{knees)}$ with conversion to total knee replacement defined as the event of interest. The number of patient knees at risk are also presented for selected time points.

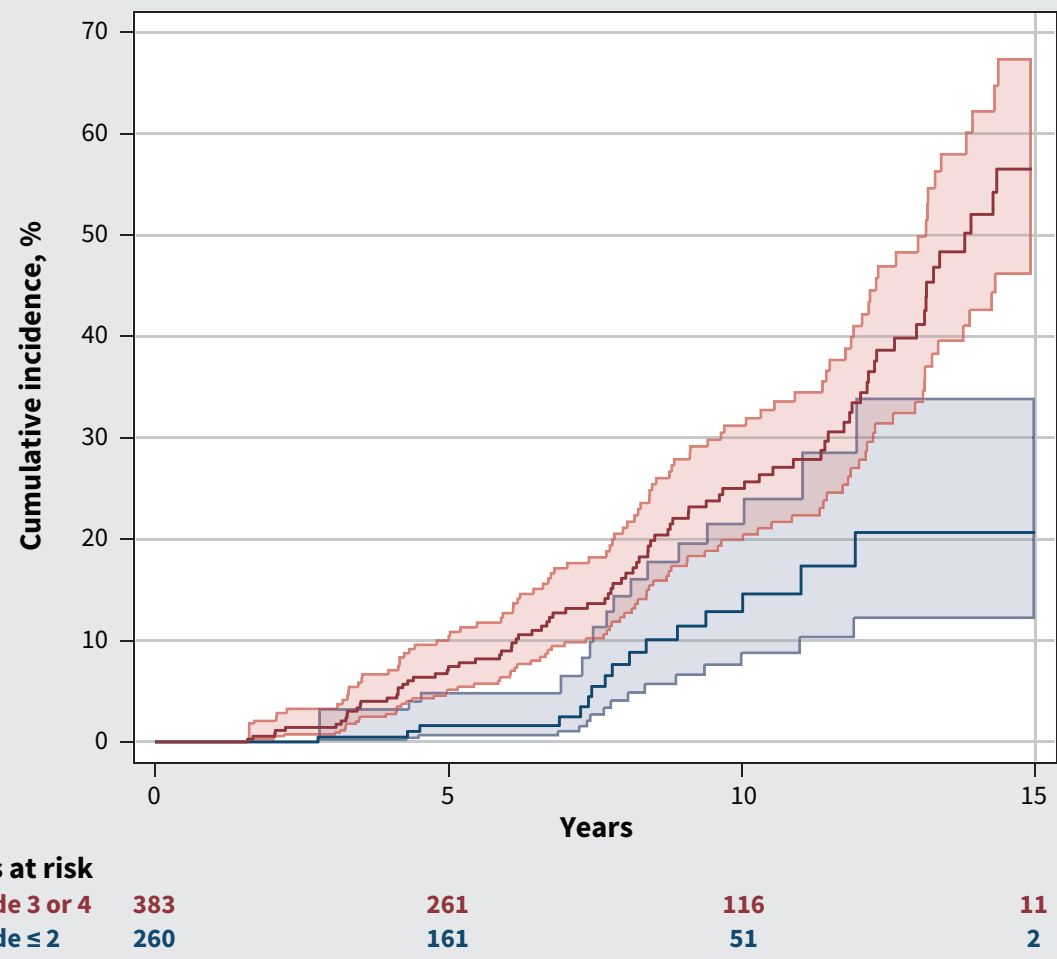

Figure 3: Cumulative incidence curve (with 95\% confidence intervals as shaded areas) for patients undergoing 1 or more high tibial osteotomies ( $n=$ 643 knees). We compared patients with mild-to-moderate radiographic disease severity (Kellgren and Lawrence grade $\leq 2 ; n=260 ;$ blue line) to those with moderate-to-severe radiographic disease severity (Kellgren and Lawrence grade 3 or $4 ; n=383$; red line), with conversion to total knee replacement defined as the event of interest. The number of patient knees at risk are also presented at selected time points for each group. 


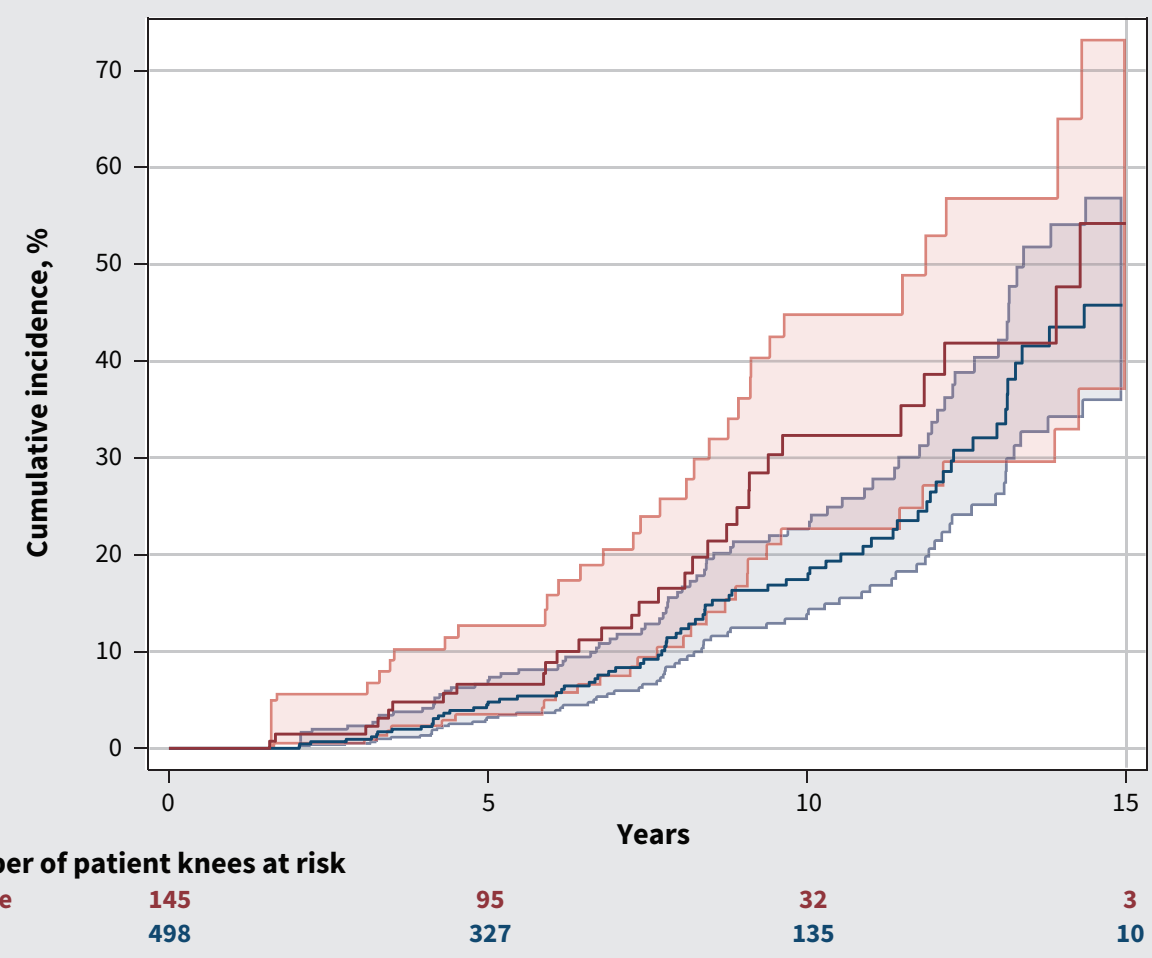

Figure 4: Cumulative incidence curve (with 95\% confidence intervals as shaded areas) for patients undergoing 1 or more high tibial osteotomies ( $n=643$ knees). We compared males ( $n=498$; blue line) to females ( $n=145$; red line), with conversion to total knee replacement defined as the event of interest. The number of patient knees at risk are also presented at selected time points for each group.

Table 2: Multivariable Cox proportional hazards estimates for predictors of conversion from high tibial osteotomy to total knee replacement

\section{Predictor}

Adjusted HR $(95 \% \mathrm{Cl})$

Radiographic severity (Kellgren and Lawrence grade) Mild to moderate* Ref. Moderate to severe*

Preoperative alignment (mechanical axis angle),

$1.05(0.98-1.11)$

Correction size, $\mathrm{mm}$

KOOS Pain subscale score (per 10 points) $\dagger$

$0.85(0.75-0.96)$

Sex

$$
\begin{aligned}
& \text { Male } \\
& \text { Female } \\
& \text { Age (per } 10 \mathrm{yr}) \\
& \text { Body mass index }\left(\text { per } 5 \mathrm{~kg} / \mathrm{m}^{2}\right) \\
& \text { Year of surgery }
\end{aligned}
$$$$
\text { Ref. }
$$$$
1.67(1.08-2.58)
$$$$
1.50(1.17-1.93)
$$$$
1.31(1.12-1.53)
$$$$
1.03(0.94-1.12)
$$

Note: $\mathrm{Cl}=$ confidence interval, $\mathrm{HR}=$ hazard ratio, $\mathrm{KOOS}=$ Knee injury and Osteoarthritis Outcome Score, Ref. = reference category. We adjusted variance for staged bilateral osteotomies using robust sandwich estimators.

*Kellgren and Lawrence grade mild to moderate $\leq 2$; moderate to severe $=3$ or 4 . †KOOS: 0 indicates extreme knee symptoms; 100 indicates no knee symptoms. with observations after lateral closing wedge HTO. ${ }^{58}$ These results might be considered obvious or biased because those patients have more time to progress to the potential need for TKR simply because they underwent HTO earlier in the disease process. However, a main rationale for targeting patients with symptomatic earlier-stage OA with HTO is secondary prevention of disease progression. ${ }^{59}$ Our findings are consistent with that goal.

Baseline symptoms (KOOS Pain subscale) were significantly associated with conversion to TKR after HTO (Table 2); we are unaware of previous studies providing comparative data. Older age, ${ }^{30,35,36,38,39}$ female sex ${ }^{30,35-38}$ and higher BMI ${ }^{46-48}$ are more commonly reported and are significantly associated with increased rate of conversion to TKR. In our study, female sex remained associated with conversion to TKR after we adjusted for other variables not previously assessed. This finding requires further investigation. Although our findings suggest a number of preoperative characteristics are statistically associated with conversion to TKR (Table 2) and should be considered in decisionmaking, they should not necessarily preclude the use of HTO. For example, our results suggest $75 \%$ of patients with $\mathrm{KL}$ grade $\geq 3$ and $68 \%$ of females do not undergo TKR within 10 years of undergoing HTO (Figure 3 and Figure 4).

Surgeons in our study used a relatively conservative surgical approach to realignment, establishing neutral-to-very-slight 
valgus rather than overcorrecting to substantial valgus. Results of the post hoc analyses were generally consistent with a decreased rate of conversion to TKR in patients with HTO corrections to $0^{\circ}-3^{\circ}$ valgus, compared with lesser or greater corrections, although the exploratory nature of those findings should be acknowledged (Appendix 2, Supplementary Tables 6 and 7).

Conversion to TKR is not the only important outcome to consider after HTO. Recent studies involving similar patients undergoing the same procedure suggest low complication rates and large, sustained, clinically important improvements in gait biomechanics, and patient-reported and performance-based outcomes after the procedure..$^{27,60}$ A 2020 systematic review and meta-analysis concluded that HTO does not negatively affect future TKR. ${ }^{61}$ Although the procedure is intended to delay OA progression and TKR, we are not aware of any published randomized controlled trials that compare HTO to competing nonoperative treatments. ${ }^{62}$

One of the strengths of our study is its prospective design that incorporated baseline variables that can be used in preoperative planning, yet are typically not possible to include in studies using administrative data. Combining prospective clinical follow-ups with clinic records enabled us to provide accurate estimates for conversion to TKR. In addition, the relatively large sample size enabled more precise estimates of cumulative incidence at 5 and 10 years. Furthermore, the number of events provided higher degrees of freedom, which allowed us to assess more predictors without the risk of overfitting the model.

\section{Limitations}

It is possible that a small number of patients sought TKR outside of London after the study censor date; therefore, the present rate of conversion from HTO to TKR should be considered within the range of previously published rates. Selection bias is also possible because patients were most often referred by other orthopedic surgeons and primary care physicians with knowledge of HTO. The patients included in our study may represent the best candidates for HTO, or those willing to participate in long-term studies, rather than all patients eligible for the procedure. The generalizability of our results may be limited to similar regions where HTO is often offered and performed. However, baseline characteristics of our sample population are similar to those reported in population-based administrative studies, and overall cumulative incidence data are only slightly different, ${ }^{30,35,36}$ which suggests that similar longevity of HTO could be observed if similar procedures were adopted at other Canadian centres. The duration of benefit from HTO may be affected by other aspects of the surgical procedure and patient characteristics that we did not assess. Finally, the decision to undergo TKR may be affected by social and geographic factors that we did not assess. ${ }^{63-65}$

\section{Conclusion}

We found that $95 \%$ of patients who undergo HTO at our centre do not go on to have TKR within 5 years, and $79 \%$ do not go on to have TKR within 10 years. The strongest predictor of conversion to TKR is greater knee OA radiographic severity at the time of HTO. These findings support the use of medial opening HTO in patients with varus alignment and media compartment knee OA to prevent or delay the need for definitive TKR.

\section{References}

1. GBD 2017 Disease and Injury Incidence and Prevalence Collaborators. Global, regional, and national incidence, prevalence, and years lived with disability for 354 diseases and injuries for 195 countries and territories, 19902017: a systematic analysis for the Global Burden of Disease Study 2017. Lancet 2018;392:1789-858.

2. Ackerman IN, Bohensky MA, Zomer E, et al. The projected burden of primary total knee and hip replacement for osteoarthritis in Australia to the year 2030. BMC Musculoskelet Disord 2019;20:90.

3. Hooper G, Lee AJ, Rothwell A, et al. Current trends and projections in the utilisation rates of hip and knee replacement in New Zealand from 2001 to 2026. N Z Med J 2014;127:82-93.

4. Singh JA, Vessely MB, Harmsen WS, et al. A population-based study of trends in the use of total hip and total knee arthroplasty, 1969-2008. Mayo Clin Proc 2010;85:898-904.

5. Vos T, Allen C, Arora M, et al. Global, regional, and national incidence, prevalence, and years lived with disability for 310 diseases and injuries, 1990-2015: a systematic analysis for the Global Burden of Disease Study 2015. Lancet 2016;388:1545-602.

6. Kurtz SM, Lau E, Ong K, et al. Future young patient demand for primary and revision joint replacement: national projections from 2010 to 2030. Clin Orthop Relat Res 2009;467:2606-12.

7. Losina E, Paltiel AD, Weinstein AM, et al. Lifetime medical costs of knee osteoarthritis management in the United States: impact of extending indications for total knee arthroplasty. Arthritis Care Res (Hoboken) 2015;67:203-15.

8. O'Donnell S, Rusu C, Hawker GA, et al. Arthritis has an impact on the daily lives of Canadians young and old: results from a population-based survey. BMC Musculoskelet Disord 2015;16:230.

9. Cross M, Smith E, Hoy D, et al. The global burden of hip and knee osteoarthritis: estimates from the global burden of disease 2010 study. Ann Rheum Dis 2014;73:1323-30.

10. Ackerman IN, Kemp JL, Crossley KM, et al. Hip and knee osteoarthritis affects younger people, too. J Orthop Sports Phys Ther 2017;47:67-79.

11. Ravi B, Croxford R, Reichmann WM, et al. The changing demographics of total joint arthroplasty recipients in the United States and Ontario from 2001 to 2007. Best Pract Res Clin Rheumatol 2012;26:637-47.

12. Hawker G, Bohm ER, Conner-Spady B, et al. Perspectives of Canadian stakeholders on criteria for appropriateness for total joint arthroplasty in patients with hip and knee osteoarthritis. Arthritis Rheumatol 2015;67:1806-15.

13. Meehan JP, Danielsen B, Kim SH, et al. Younger age is associated with a higher risk of early periprosthetic joint infection and aseptic mechanical failure after total knee arthroplasty. J Bone Joint Surg Am 2014;96:529-35.

14. Bourne RB, Chesworth BM, Davis AM, et al. Patient satisfaction after total knee arthroplasty: Who is satisfied and who is not? Clin Orthop Relat Res 2010;468:57-63.

15. Bayliss LE, Culliford D, Monk AP, et al. The effect of patient age at intervention on risk of implant revision after total replacement of the hip or knee: a population-based cohort study. Lancet 2017;389:1424-30.

16. Dy CJ, Marx RG, Bozic KJ, et al. Risk factors for revision within 10 years of total knee arthroplasty. Clin Orthop Relat Res 2014;472:1198-207.

17. Julin J, Jamsen E, Puolakka T, et al. Younger age increases the risk of early prosthesis failure following primary total knee replacement for osteoarthritis. A follow-up study of 32019 total knee replacements in the Finnish Arthroplasty Register. Acta Orthop 2010;81:413-9.

18. Paterson JM, Williams JI, Kreder HJ, et al. Provider volumes and early outcomes of primary total joint replacement in Ontario. Can J Surg 2010;53:175-83.

19. Ghomrawi HMK, Mushlin Al, Kang R, et al. Examining timeliness of total knee replacement among patients with knee osteoarthritis in the US: results from the OAI and MOST longitudinal cohorts. J Bone Joint Surg Am 2020;102:468-76. 
20. Li CS, Karlsson J, Winemaker M, et al. Orthopedic surgeons feel that there is a treatment gap in management of early OA: international survey. Knee Surg Sports Traumatol Arthrosc 2014;22:363-78.

21. London NJ, Miller LE, Block JE. Clinical and economic consequences of the treatment gap in knee osteoarthritis management. Med Hypotheses 2011;76:887-92.

22. Hui C, Thompson SR, Giffin JR. Knee arthritis. In: Miller MD, Thompson SR, editors. DeLee \& Drez's orthopaedic sports medicine textbook, 4th ed. New York: Elsevier; 2014:1227-42.

23. McNamara I, Birmingham TB, Fowler PJ, et al. High tibial osteotomy: evolution of research and clinical applications - a Canadian experience. Knee Surg Sports Traumatol Arthrosc 2013;21:23-31.

24. Leitch KM, Birmingham TB, Dunning CE, et al. Changes in valgus and varus alignment neutralize aberrant frontal plane knee moments in patients with unicompartmental knee osteoarthritis. J Biomech 2013;46:1408-12.

25. Leitch KM, Birmingham TB, Dunning CE, et al. Medial opening wedge high tibial osteotomy alters knee moments in multiple planes during walking and stair ascent. Gait Posture 2015;42:165-71.

26. Birmingham TB, Giffin JR, Chesworth BM, et al. Medial opening wedge high tibial osteotomy: a prospective cohort study of gait, radiographic, and patientreported outcomes. Arthritis Rheum 2009;61:648-57.

27. Birmingham TB, Moyer R, Leitch $\mathrm{K}$, et al. Changes in biomechanical risk factors for knee osteoarthritis and their association with 5-year clinically important improvement after limb realignment surgery. Osteoarthritis Cartilage 2017;25:1999-2006

28. Konopka JF, Gomoll AH, Thornhill TS, et al. The cost-effectiveness of surgical treatment of medial unicompartmental knee osteoarthritis in younger patients: a computer model-based evaluation. J Bone Joint Surg Am 2015;97:807-17.

29. Smith WB II, Steinberg J, Scholtes S, et al. Medial compartment knee osteoarthritis: age-stratified cost-effectiveness of total knee arthroplasty, unicompartmental knee arthroplasty, and high tibial osteotomy. Knee Surg Sports Traumatol Arthrosc 2017;25:924-33.

30. Khoshbin A, Sheth $U$, Ogilvie-Harris D, et al. The effect of patient, provider and surgical factors on survivorship of high tibial osteotomy to total knee arthroplasty: a population-based study. Knee Surg Sports Traumatol Arthrosc 2017;25:887-94.

31. Degen RM, Lebedeva Y, Birmingham TB, et al. Trends in knee arthroscopy utilization: a gap in knowledge translation. Knee Surg Sports Traumatol Arthrosc 2020;28:439-47.

32. Hip and knee replacements in Canada, 2017-2018: Canadian Joint Replacement Registry annual report. Ottawa: Canadian Institute for Health Information; 2019.

33. Dhawan A, Mather RC III, Karas V, et al. An epidemiologic analysis of clinical practice guidelines for non-arthroplasty treatment of osteoarthritis of the knee. Arthroscopy 2014;30:65-71.

34. Wright J, Heck D, Hawker G, et al. Rates of tibial osteotomies in Canada and the United States. Clin Orthop Relat Res 1995;(319):266-75.

35. W-Dahl A, Robertsson O, Lohmander LS. High tibial osteotomy in Sweden, 1998-2007: a population-based study of the use and rate of revision to knee arthroplasty. Acta Orthop 2012;83:244-8.

36. Niinimäki TT, Eskelinen A, Mann BS, et al. Survivorship of high tibial osteotomy in the treatment of osteoarthritis of the knee: Finnish registry-based study of 3195 knees. J Bone Joint Surg Br 2012;94:1517-21.

37. Keenan OJF, Clement ND, Nutton R, et al. Older age and female gender are independent predictors of early conversion to total knee arthroplasty after high tibial osteotomy. Knee 2019;26:207-12.

38. van Wulfften Palthe AFY, Clement ND, Temmerman OPP, et al. Survival and functional outcome of high tibial osteotomy for medial knee osteoarthritis: a 10-20-year cohort study. Eur J Orthop Surg Traumatol 2018;28:1381-9.

39. Trieb K, Grohs J, Hanslik-Schnabel B, et al. Age predicts outcome of high-tibial osteotomy. Knee Surg Sports Traumatol Arthrosc 2006;14:149-52.

40. Fowler PJ, Tan JL, Brown GA. Medial opening wedge high tibial osteotomy: How I do it. Oper Tech Sports Med 2012;20:87-92.

41. Specogna AV, Birmingham TB, Hunt MA, et al. Radiographic measures of knee alignment in patients with varus gonarthrosis: effect of weightbearing status and associations with dynamic joint load. Am J Sports Med 2007; 35:65-70.
42. Specogna AV, Birmingham TB, DaSilva JJ, et al. Reliability of lower limb frontal plane alignment measurements using plain radiographs and digitized images. J Knee Surg 2004;17:203-10.

43. Lau B, Cole SR, Gange SJ. Competing risk regression models for epidemiologic data. Am J Epidemiol 2009;170:244-56.

44. Kellgren JH, Lawrence JS. Radiological assessment of osteo-arthrosis. Ann Rheum Dis 1957;16:494-502.

45. Roos EM, Roos HP, Lohmander LS, et al. Knee injury and osteoarthritis outcome Score (KOOS) - development of a self-administered outcome measure. J Orthop Sports Phys Ther 1998;28:88-96.

46. Akizuki S, Shibakawa A, Takizawa T, et al. The long-term outcome of high tibial osteotomy. J Bone Joint Surg Br 2008;90:592-6.

47. Flecher X, Parratte S, Aubaniac J-M, et al. 12-28-year follow-up study of closing wedge high tibial osteotomy. Clin Orthop Relat Res 2006;(452):91-6.

48. Naudie D, Bourne RB, Rorabeck CH, et al. The Install Award. Survivorship of the high tibial valgus osteotomy. A 10- to -22-year follow-up study. Clin Orthop Relat Res 1999;(367):18-27.

49. Babyak MA. What you see may not be what you get: a brief, nontechnical introduction to overfitting in regression-type models. Psychosom Med 2004;66:411-21.

50. Altman R, Asch E, Bloch D, et al. Development of criteria for the classification and reporting of osteoarthritis. Classification of osteoarthritis of the knee. Diagnostic and Therapeutic Criteria Committee of the American Rheumatism Association. Arthritis Rheum 1986;29:1039-49.

51. Bode G, von Heyden J, Pestka J, et al. Prospective 5-year survival rate data following open-wedge valgus high tibial osteotomy. Knee Surg Sports Traumatol Arthrosc 2015;23:1949-55.

52. Bonasia DE, Dettoni F, Sito G, et al. Medial opening wedge high tibial osteotomy for medial compartment overload/arthritis in the varus knee: prognostic factors. Am J Sports Med 2014;42:690-8.

53. Schuster $\mathrm{P}$, Schulz $\mathrm{M}$, Mayer $\mathrm{P}$, et al. Open-wedge high tibial osteotomy and combined abrasion/microfracture in severe medial osteoarthritis and varus malalignment: 5-year results and arthroscopic findings after 2 years. Arthroscopy 2015;31:1279-88.

54. Woodacre T, Ricketts M, Evans JT, et al. Complications associated with opening wedge high tibial osteotomy - a review of the literature and of 15 years of experience. Knee 2016;23:276-82.

55. Ekeland A, Nerhus TK, Dimmen S, et al. Good functional results following high tibial opening-wedge osteotomy of knees with medial osteoarthritis: a prospective study with a mean of 8.3 years of follow-up. Knee 2017;24:380-9.

56. Duivenvoorden T, van Diggele P, Reijman M, et al. Adverse events and survival after closing- and opening-wedge high tibial osteotomy: a comparative study of 412 patients. Knee Surg Sports Traumatol Arthrosc 2017;25:895-901.

57. Hernigou P, Ma W. Open wedge tibial osteotomy with acrylic bone cement as bone substitute. Knee 2001;8:103-10.

58. Efe T, Ahmed G, Heyse TJ, et al. Closing-wedge high tibial osteotomy: survival and risk factor analysis at long-term follow up. BMC Musculoskelet Disord 2011;12:46. doi: 10.1186/1471-2474-12-46.

59. Primeau CA, Birmingham TB, Leitch KM, et al. Degenerative meniscal tears and high tibial osteotomy: Do current treatment algorithms need to be realigned? Clin Sports Med 2019;38:471-82.

60. Martin R, Birmingham TB, Willits K, et al. Adverse event rates and classifications in medial opening wedge high tibial osteotomy. Am J Sports Med 2014;42:1118-26.

61. Sun X, Wang J, Su Z. A meta-analysis of total knee arthroplasty following high tibial osteotomy versus primary total knee arthroplasty. Arch Orthop Trauma Surg 2020;140:527-35.

62. Brouwer RW, Huizinga MR, Duivenvoorden T, et al. Osteotomy for treating knee osteoarthritis. Cochrane Database Syst Rev 2007;(3):CD004019.

63. Hawker GA, Guan J, Croxford R, et al. A prospective population-based study of the predictors of undergoing total joint arthroplasty. Arthritis Rheum 2006;54:3212-20.

64. Kwoh CK, Vina ER, Cloonan YK, et al. Determinants of patient preferences for total knee replacement: African-Americans and whites. Arthritis Res Ther 2015;17:348.

65. Hawker GA, Wright JG, Coyte PC, et al. Differences between men and women in the rate of use of hip and knee arthroplasty. N Engl J Med 2000;342:1016-22. 
Competing interests: Trevor Birmingham, Bert Chesworth, Dianne Bryant and J. Robert Giffin have received grants from the Canadian Institutes of Health Research and The Arthritis Society during the conduct of the study. Kevin Willits has received grants from Smith \& Nephew outside of the submitted work. Robert Litchfield has received personal fees from ConMed Linvatec, Smith \& Nephew, DePuy and Arthrosurface, and grants from Smith \& Nephew and DePuy outside the submitted work. No other competing interests were declared.

This article has been peer reviewed.

Affiliations: Fowler Kennedy Sport Medicine Clinic (Primeau, Birmingham, Leitch, Willits, Litchfield, Fowler, Bryant, Giffin); School of Physical Therapy, Faculty of Health Sciences (Primeau, Birmingham, Marsh, Chesworth, Bryant); Collaborative Specialization in Musculoskeletal Health Research, Bone and Joint Institute (Primeau); Bone and Joint Institute (Birmingham, Willits, Litchfield, Marsh, Chesworth, Bryant, Giffin); Department of Surgery, Schulich School of Medicine and Dentistry (Willits, Litchfield, Giffin); Department of
Epidemiology and Biostatistics (Dixon), Western University; Lawson Health Research Institute (Dixon), London, Ont.

Contributors: Codie Primeau, Trevor Birmingham, Jacquelyn Marsh, Bert Chesworth, Dianne Bryant and J. Robert Giffin contributed to conception and design of the work. Codie Primeau, Kristyn Leitch, Kevin Willits, Robert Litchfield, Peter Fowler and J. Robert Giffin contributed to data acquisition. Codie Primeau, Trevor Birmingham, Jacquelyn Marsh, Bert Chesworth, Stephanie Dixon and J. Robert Giffin contributed to data analysis and interpretation of the results. Codie Primeau and Trevor Birmingham drafted the manuscript; all of the other authors revised the manuscript critically for important intellectual content. All of the authors gave final approval of the version to be published and agreed to be accountable for all aspects of the work.

Content licence: This is an Open Access article distributed in accordance with the terms of the Creative Commons Attribution (CC BY-NC-ND 4.0) licence, which permits use, distribution and reproduction in any medium, provided that the original publication is properly cited, the use is noncommercial (i.e., research or educational use), and no modifications or adaptations are made. See: https://creativecommons.org/ licenses/by-nc-nd/4.0/

Funding: This research was undertaken, in part, thanks to the funding from the Canadian Institutes of Health Research; The Arthritis Society, Canada; the Canadian Research Chairs Program(TBB); and a Transdisciplinary Training Award to Codie Primeau from the Bone and Joint Institute at Western University.

Data sharing: Unidentified study data may be made available to other researchers upon request to the corresponding authors and with completion of data-sharing agreements.

Disclaimer: All of the authors were independent of the funders. Funders were not involved with study design, data collection, analysis or interpretation of the data, or writing of the manuscript.

Accepted: Nov. 10, 2020

Correspondence to: Trevor Birmingham tbirming@uwo.ca; J. Robert Giffin, rgiffin@uwo.ca 